\title{
Vibro-impact response of a cracked bar
}

\author{
Vikrant R. Hiwarkar*, Vladimir I. Babitsky and Vadim V. Silberschmidt \\ Wolfson School of Mechanical and Manufacturing Engineering, Loughborough University, Loughborough, \\ Leicestershire, LE11 3TU, UK
}

Received 12 February 2010

Revised 25 May 2010

\begin{abstract}
The presence of a crack in a structure affects its dynamic behaviour under working conditions. Cracks introduce nonlinearities into the system; the use of such nonlinearities for damage detection should be investigated. A model of a onedimensional cracked cantilever bar subjected to longitudinal harmonic excitation is used to analyse a vibro-impact response as a way to monitor structural health. The effect of contact nonlinearity due to crack's faces interaction is considered. This nonlinear information is obtained based on a combination of the analytical technique and the Matlab-Simulink computation. The procedure uses a numerical approximation for dynamic compliance operators and a nonlinear model of contact faces interaction implemented numerically as a nonlinear feedback. Nonlinear resonant phenomena due to vibro-impact interaction in the cracked bar are obtained and analysed. A distribution of the higher harmonics along the bar length, generated due to the nonlinear response of the crack, is revealed as a function of the distance from the crack. Recommendations on structural health monitoring of cracked bars due to contact nonlinearity are presented
\end{abstract}

Keywords: Vibro-impact, nonlinear vibration, crack monitoring

\section{Introduction}

Crack monitoring in critical structures is becoming more important to prevent failures occurring under operating conditions. In most of the cases the failure occurs due to the initiation and growth of a crack. As a result of this there has been huge losses faced by the power plant, aerospace and other industry. Hence it becomes necessary to monitor the health of structures and components under operating condition. At present there are many structural health monitoring techniques. But most of the techniques are used for a specific purpose and some of techniques require the structure to be dismantled for testing making procedure of non-destructive testing too much expensive and time consuming. So there arises a need for new more cost effective techniques that can be used for monitoring structural health continuously. So far several researchers have addressed the problem of a beam with the breathing cracks using the finite element method and validated the results obtained through experimental analysis. In finite element approach, additional flexibility due to the crack was evaluated using the strain energy density function given by a linear fracture mechanics theory. Based on this flexibility an element stiffness matrix was deduced for finite element analysis [1,2]. The finite element model for the cracked beam was also suggested by integrating the stress intensity factor to obtain the element stiffness matrix [3]. In that finite element analysis considering the flexibility due to crack it was found that the resonant frequencies and the vibration amplitude were considerably affected by the existence of the crack. In [4] the beam with a transverse crack was modelled using the triangular disk finite element. The location of the crack in that approach was determined by the deflection shape of the beam it was found that the amplitude of vibration was three times higher than in the case of vibration without a crack. An approximate Galerkin solution to one dimensional beam theory was developed in [5] for free bending beams with pairs of symmetric open cracks [6]. That approach was useful to determine the higher natural frequency and the mode

\footnotetext{
*Corresponding author. Tel.: +44 1509 227566; Fax: +44 1509 227615; E-mail: v.hiwarkar@lboro.ac.uk.
} 
shape of the cracked beam. The results obtained predicted the changes in eigen frequencies and eigen modes due to cracks. The finite-element model of a beam with a breathing crack subjected to harmonic loading was studied by solving the nonlinear algebraic equations numerically [7]. In [8] a special finite element was developed for a cracked Timoshenko beam using shape functions for rotational and transverse displacements to obtain a consistent matrix for the cracked beam element. In this way the effect of the crack on the stiffness matrix and consistent matrix was investigated. Further, a finite-element model of a cracked beam that was coupled with identification methodology may be useful for detecting the cracks in engineering applications. A two dimensional finite-element analysis was performed for a cracked cantilever beam under harmonic loading [9]. It was found that its frequency did not change with oscillation amplitude but the steady state response obtained had multiple sub-harmonic and super- harmonic components. It was also found that when the forcing frequency coincided with or was integer sub-multiples of the first system frequency $\omega_{0}$, then the $n$-th harmonic component frequency of the forcing frequency, which was close to $\omega_{0}$ would be significantly exalted. Moreover, within the super-harmonic resonance ranges the phase portraits were characterized by significant wiggles due to impact between crack faces. So far few researchers addressed the problem of breathing cracks by modelling them as a simple spring mass system. In [10] the transverse vibrations of a cracked beam were studied with time varying stiffness. The equation of motion was solved by employing a harmonic balance technique. It was shown that, when the breathing crack is present, higher harmonics in frequency were generated revealing the nonlinear behaviour of the system. The nonlinear behaviour of a beam with a closing crack, vibrating in its first mode of vibration, through a simple single degree of freedom was modelled with bilinear stiffness [11]. The response due to harmonic excitation was obtained using numerical integration. Integral multiples of the forcing frequencies were found in the frequency spectrum. In [12] a modal analysis was used to study the nonlinear dynamics of a cantilever bar with a transverse crack edge. In that work, the crack was modelled as a piecewise linear system. In order to understand essential nonlinear dynamics of the cracked beam, a piecewise linear two degree of freedom model was studied. In [13] the beam was modelled as an oscillator with a bilinear restoring force and applied the bi-spectral analysis to the response. The analysis showed high sensitivity to the non-linear behaviour of the system compared to other techniques. In [14] the finite-element simulation was used to study the longitudinal harmonic loading for assessing damage in a crack cantilever bar. The excitation resulted in the opening and closing of the crack considered in simulation. For nonlinear materials their properties were introduced in terms of the plastic behaviour. The effect of the crack size and position along the beam length was studied. An observed change in the frequency response can be used to characterize the damage state of the component.

In this paper the nonlinear resonant phenomena due to vibro-impact interaction within a cracked bar is studied with the novel technique of simulation using Matlab-Simulink. The presence of a crack in a structure is treated like a discontinuity which affects the dynamic behaviour and results in generation of significant additional perturbations. The discontinuous element in continuous structure is modelled using a special mathematical technique for proper matching of local and distributed elements of the resulting structure. This is effectively implemented using the force characteristics of contact interaction for the discontinuous elements and Green's function in the contact areas for the media [15]. This description generally leads to application of integral equation. Laplace transformation of the integral equation produce operators for the following simulation of the interaction, where local discontinuity is implanted as a nonlinear feedback and continuous elements as the operators of dynamic compliance (receptance) of the media at the contact points. Effective numerical simulation of such structures with Matlab-Simulink software requires implementation of realistic models for contact phenomena and transformation of the transcendental operators of dynamic compliances into rational functions of complex variable. This is made with the help of modal representation for the dynamic compliances. Uniform convergence of the modal approximation leads to high accuracy of simulation within the use of first few initial modes. This also produces a convenient calculation scheme for accurate estimation.

\section{Problem formulation}

Consider a cantilever bar of length $l$ with a plane cross-sectional area $S$ excited by the harmonic force $P(l, t)=$ $a_{p} \cos \omega t$. The crack is considered at the free end of the bar and is modelled as a limiter stop with a distance from the free end (Fig. 1). Under the application of the harmonic force the crack's faces interact. This interaction of the bar with the limiter (considered as a crack in this case) leads to generation of the nonlinear contact force due 


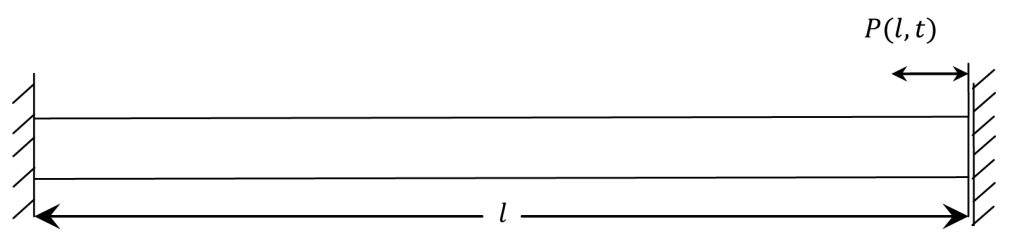

Fig. 1. Schematic of cantilever bar with crack at the free end (modelled as limiter).

to opening and closing of the crack. The concept of dynamic compliance and the contact force characteristics of impact interaction are considered for analysing the dynamics of the bar due to the crack [15]. The blocks of the dynamic compliance and the contact force were modelled with Matlab-Simulink.

\section{Equation of motion of cantilever bar}

Consider the bar having the modulus of elasticity $E$ and density $\rho$ as shown in Fig. 1. The absolute displacement for arbitrary section is taken as $u(x, t)$ under the application of a harmonic force $P(l, t)=a_{p} \cos \omega t$. The differential equation Eq. (1) for the longitudinal vibration of a uniform rod without the internal damping of the material is

$$
\frac{\partial^{2} u(x, t)}{\partial t^{2}}=\gamma^{2} \frac{\partial^{2} u(x, t)}{\partial x^{2}}
$$

where

$$
\gamma=\sqrt{E / \rho}
$$

Internal damping is common to range of mechanisms that occur in the deformation of the materials that are cyclically strained; in particular, internal damping is the cause of hysteresis loops that occur in the stress/strain curve. Several experimental results have shown that under a periodic deformation the area of hysteresis loops for most materials depends on the amplitude of deformation for a wide range of change but not on frequency. For this reason, in the study of a periodic process an equivalent force, proportional to the velocity of deformation, is introduced selecting a suitable factor so that the general dissipative effect corresponds to the experimental data [16]. As a result, Eq. (1) for the study of periodic vibration of the rod with the internal damping can be written in the following form

$$
\frac{\partial^{2} u(x, t)}{\partial t^{2}}-\gamma^{2} \frac{\partial^{2} u(x, t)}{\partial x^{2}}-b \frac{\partial^{3} u(x, t)}{\partial x^{2} \partial t}=0
$$

where $b=\gamma^{2} \chi / 2 \pi \omega$ is the linearised coefficient of force of internal damping; $\omega$ is the frequency of excitation; $\chi$ is the absorbtion coefficient found from the test as the ratio of energy absorbtion during the cycle (proportional to the hysteresis loop) to the basic deformation energy. Because of internal damping, the periodic process can only be established in a case with a regular addition of energy into the system.

\section{Expression of dynamic compliance of cantilever bar}

Following [15], the expression for the dynamic compliance (receptance) can be derived by applying the boundary condition of the cantilever bar to the Eq. (2).

Fixed End:

$$
u(0, t)=0 ;
$$

Free End:

$$
E S\left(1+\frac{b}{\gamma^{2}}\right)\left[\frac{\partial u(x, t)}{\partial x}\right]_{x=l}=\exp (j \omega t) .
$$

Assuming a solution in the form 


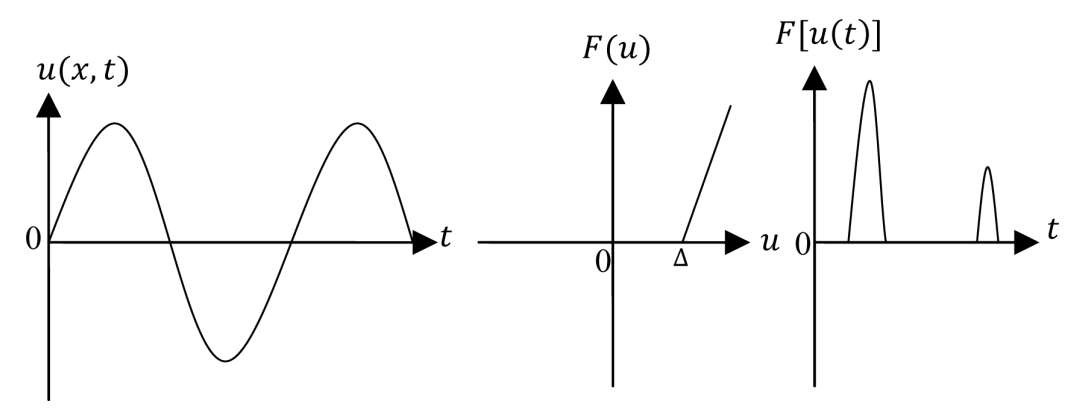

Fig. 2. Contact force characteristics.

$$
u(x, t)=u_{x}(t)=L_{l}(x, j \omega) \exp (j \omega t),
$$

where $L_{l}(x, j \omega)$ is a receptance coupling for the force acting at and displacement at co-ordinate

Substituting Eq. (5) to Eq. (2), Eqs (3) and (4) we have

$$
\begin{aligned}
& (j \omega)^{2} L_{l}(x, j \omega)-\left(\gamma^{2}+j \omega b\right) \frac{d^{2} L_{l}(x, j \omega)}{d x^{2}}=0 \\
& L_{l}(0, j \omega)=0, \\
& E S\left(1+j \omega \frac{b}{\gamma^{2}}\right)\left[\frac{d L_{l}(x, j \omega)}{d x}\right]_{x=l}=1
\end{aligned}
$$

The solution of differential Eq. (6) of the second order with constant coefficients satisfying the boundary conditions Eqs (7) and (8) leads to the following expression for dynamic compliance.

$$
L_{l}(l, j \omega)=\frac{l}{E S \zeta}\left[\tan \zeta-j \frac{\chi}{4 \pi} \frac{\zeta+1 / 2 \sin 2 \zeta}{\cos ^{2} \zeta}\right]
$$

where

$$
\xi=\frac{\omega l}{\gamma} .
$$

\section{Characteristics of contact force}

Under harmonic loading the bar interacts with the limiter (Fig. 1). This interaction with the limiter transforms the continuous process into successive impulses modulated by the velocity of the input process at the instant when the process reaches the threshold value. Such nonlinear components of the structural scheme are called the impact elements [15]. These impact elements have positional impulse effects, which specifically combine the effects of the relayed and impulse elements. Figure 2 shows an example of transformations of deformation into the force by impact elements. This force characteristic of the contact interaction is given by an expression called static force characteristics of the impact pair [15]:

$$
F(u)=\psi(x-\Delta) \eta(x-\Delta)
$$

where

$$
\eta(x)=\left\{\begin{array}{l}
0, x<0 \\
1, x \geqslant 0
\end{array}\right.
$$




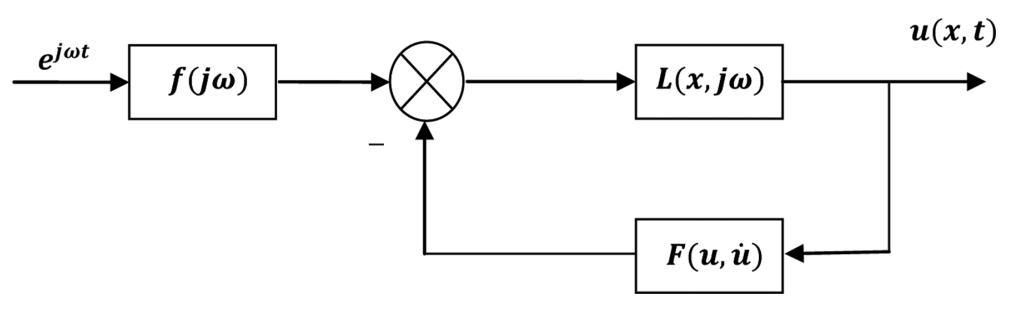

Fig. 3. Block diagram.

\section{Dynamic model of cracked cantilever bar}

From the schematic shown in Fig. 1 it is clear that when the harmonic loading is acting on the bar it starts to interact with a limiter, generating the contact force that affects deformation of the bar. This contact force generated is implanted as the nonlinear feedback as shown in Fig. 3. When the cantilever bar interacts with the limiter, the vibrational displacement of an arbitrary section $x$ is defined as a function of $u(x, t)$. By introducing the contact force characteristics $F(u, \dot{u})$ and dynamic compliance operator $L(x, j \omega)$, coupling displacement $u(x, t)$ to the force acting at $x=l$, the operator equation for the unknown function $u(x, t)$ can be written in the following form:

$$
u(x, t)=L_{l}(x, s) P(l, t)-L_{l}(x, s) F(u, \dot{u})
$$

The closed form of dynamic compliance in Eq. (9) cannot be implemented numerically. For straight rods undergoing axial vibration this can be implemented by splitting it into infinite number of modes [17]. The dynamic compliance of the cross section $x=p$ under the force applied to cross section $x=q$ for the straight elastic rods undergoing axial vibration can be written as [15]:

$$
L_{p q}(s)=\sum_{v=1}^{1} \frac{A_{v}(p) A_{v}(q)}{s^{2}+2 r_{v} \Omega_{v} s+\Omega_{v}^{2}},
$$

in which $s$ is a complex variable. The mechanism of energy dissipation in Eq. (13) is represented in the form of internal damping in the material, given by $r_{v}=\frac{\chi \Omega_{v}}{4 \pi \omega}$. Here $\chi$ represents an absorption coefficient, $\Omega_{v}$ represents the natural frequency and $\omega$ is the frequency of excitation. The number of vibration modes in Eq. (13) increases infinitely and the coefficients for each mode remains functions of continuous coordinate $x$, which represents the position of section under consideration; these coefficients are denoted as $A_{v}(x)$. It is also called the modal shape function.

The simulation with Matlab-Simulink is performed on the cantilever bar taking Eqs (12) and (13) into account. For simulation purpose only first three modes of vibration are taken into consideration which gives the accurate numerical results. For simulations the material properties of steel rod are considered having dimensions $300 \mathrm{~mm} \times$ $25 \mathrm{~mm} \times 10 \mathrm{~mm}$.

\section{Response of cantilever bar without crack (Limiter)}

Initially, the forced longitudinal vibration was performed on a cantilever bar without crack. The frequency sweep was performed in the Matlab-Simulink model with frequencies ranging from $3500 \mathrm{~Hz}$ to $30000 \mathrm{~Hz}$. The results in Fig. 4(a) and (b) show the time and frequency response, respectively, of the cantilever bar. From the time response it can be observed that it is symmetric about the $\mathrm{x}$-axis. The frequency response of the system clearly shows that the resonance occurring at the frequencies of $4206 \mathrm{~Hz}, 12620 \mathrm{~Hz}$ and $21029 \mathrm{~Hz}$.

\section{Response of cantilever bar with crack (Limiter)}

Similarly, for a cantilever bar with a crack, the frequency sweep was performed from $3500 \mathrm{~Hz}$ to $30000 \mathrm{~Hz}$ in the Matlab-Simulink model. The result in Fig. 5(a) and (b) shows the time response and frequency response, 


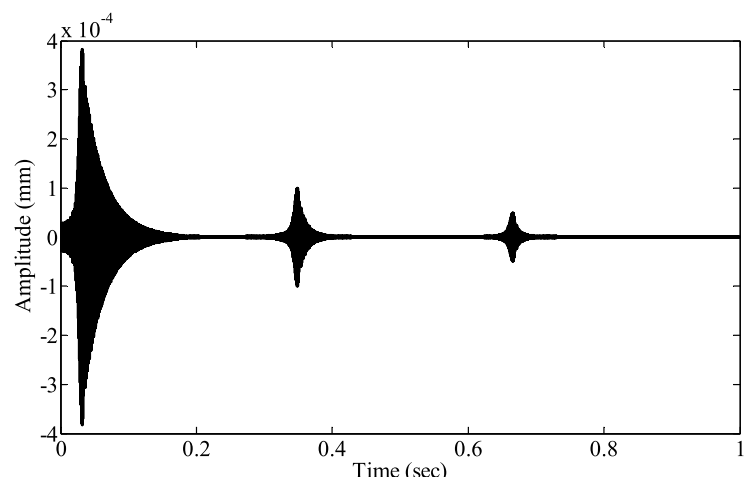

(a) Time response

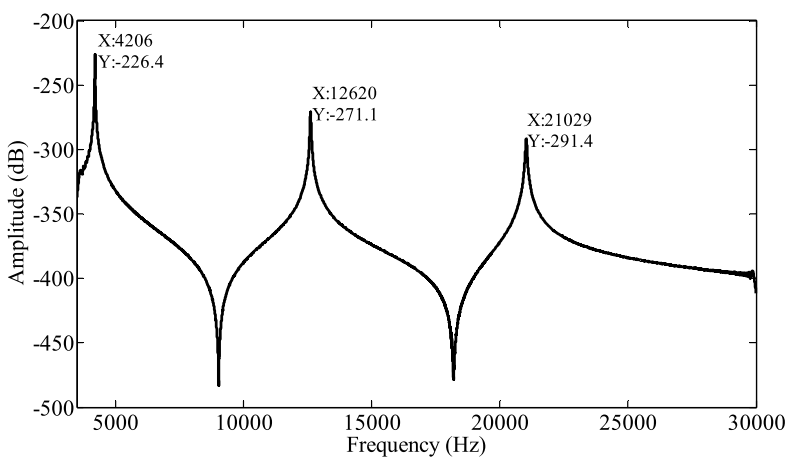

(b) Frequency response

Fig. 4. Response of cantilever bar (linear case).

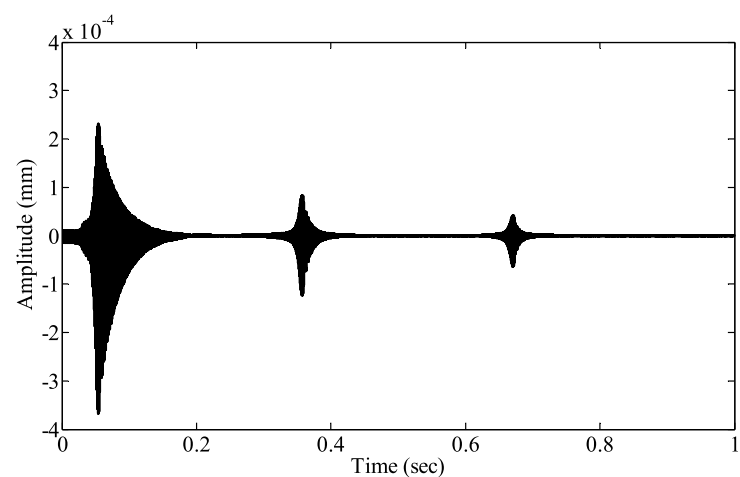

(a) Time response

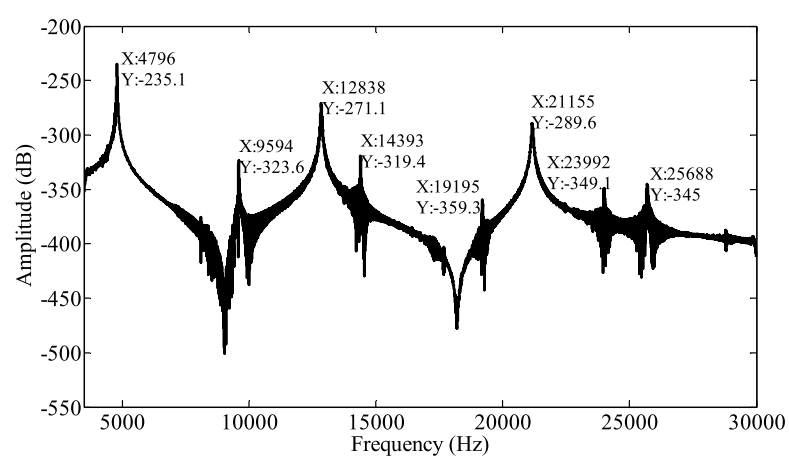

(b) Frequency response

Fig. 5. Response of cracked cantilever bar (nonlinear case).

respectively. From the time response it can be observed that the time signal has lost its symmetry, indicating distortion. This distortion in time signal is an indication of nonlinearity due to opening and closing of the crack. In frequency response (Fig. 5(b)) it can be observed that there has been shift in the frequency when compared with the frequency response of the uncracked bar (Fig. 4(b)) along with higher harmonic frequency components occurring in the frequency response indicating the crack-induced nonlinearity.

\section{Effect of linear resonance frequency on cracked bar}

For the cantilever bar without a crack the first three resonances frequency occurs at $4206 \mathrm{~Hz}, 12620 \mathrm{~Hz}$ and $21029 \mathrm{~Hz}$. The cracked bar was excited at each of these resonance frequencies of the uncracked bar to obtain the steady-state response; the results are presented in Figs 6,7 and 8. It can be observed that modulation in the time signal indicates the presence of some additional frequency. From frequency response it is clear that along with the frequency of excitation there is an additional frequency component coupled with it. These additional frequency components are caused by free vibration taking place during the process of forced excitation, i.e. the interaction between the cantilever bar and the limiter.

\section{Effect of nonlinear resonance frequency on cracked bar}

For the cantilever bar with a crack it can be seen in its frequency response (Fig. 5(b)) that a nonlinear resonance occurs at the frequencies $4796 \mathrm{~Hz}, 12838 \mathrm{~Hz}$ and $21155 \mathrm{~Hz}$. When the cracked cantilever bar is excited at the 


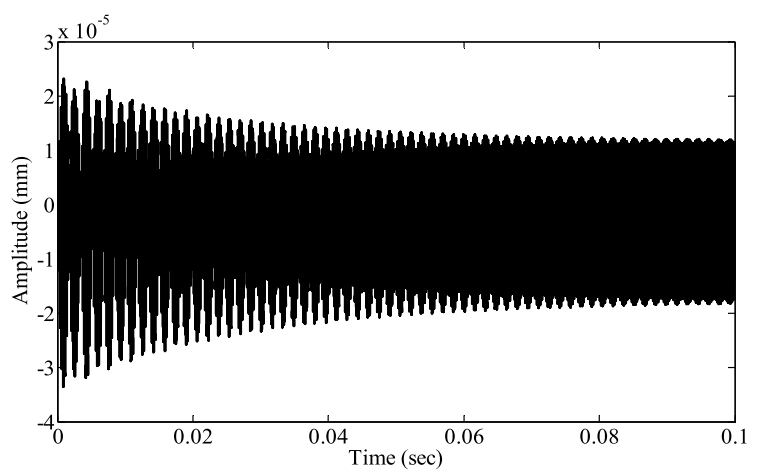

(a) Time response

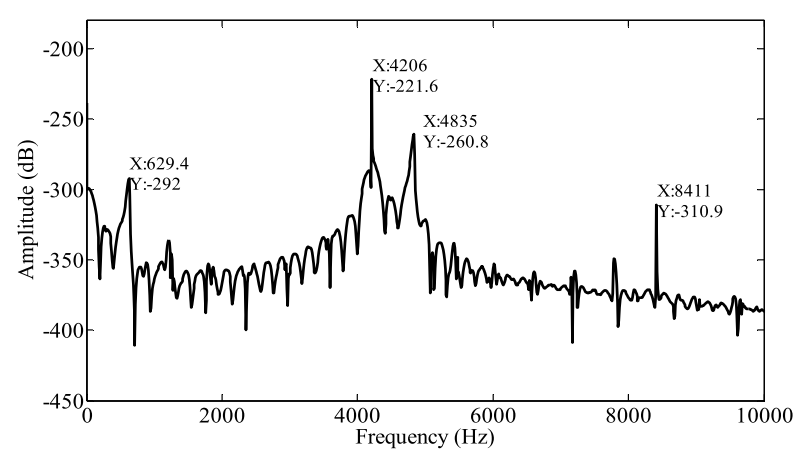

(b) Frequency response

Fig. 6. Effect of first linear resonance frequency of excitation on cracked bar.

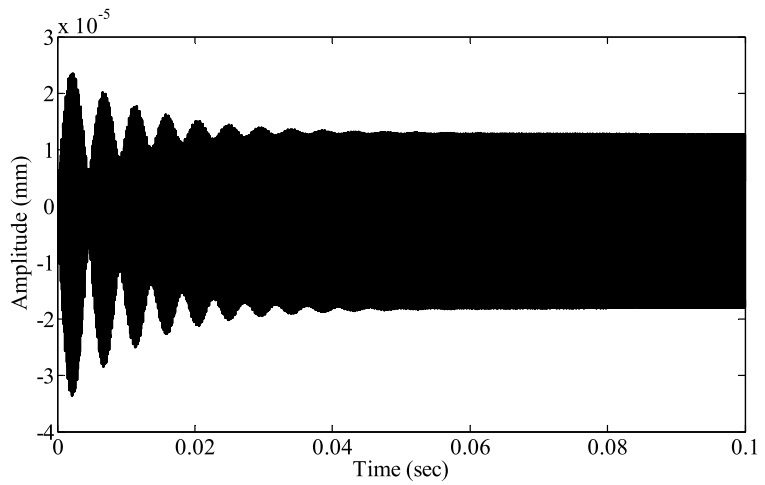

(a) Time response

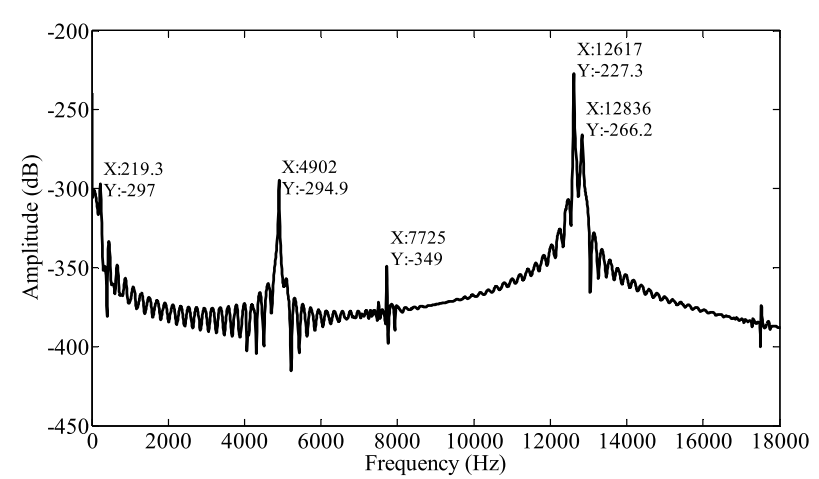

(b) Frequency response

Fig. 7. Effect of second linear resonance frequency of excitation on cracked bar.

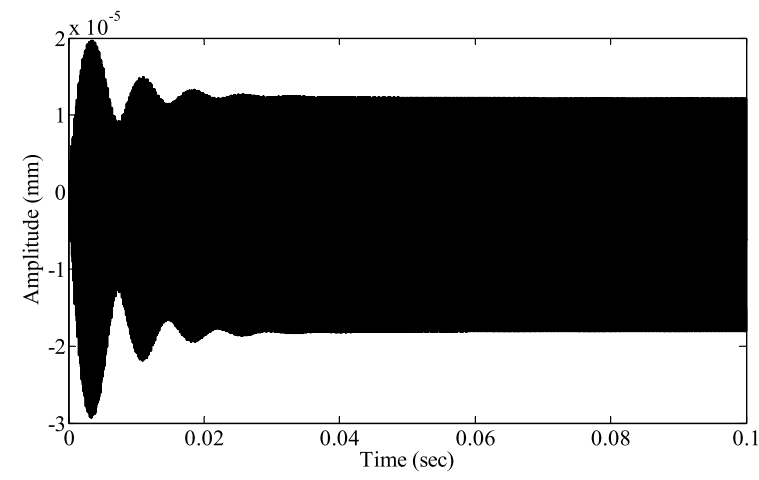

(a) Time response

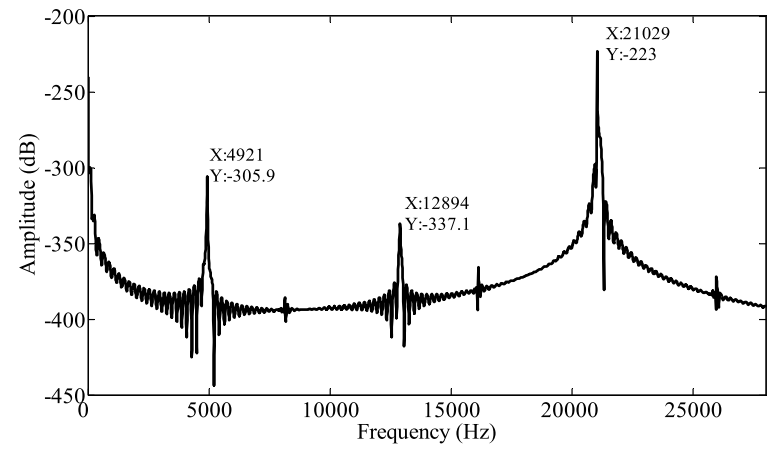

(b) Frequency response

Fig. 8. Effect of third linear resonance frequency of excitation on cracked bar.

nonlinear resonant frequency its time responses (Figs 9(a), 10(a) and 11(a)) obtained show no modulation of the signal indicating only the presence of one frequency at the steady-state vibration. This can be clearly seen from the frequency response (Figs 9(b), 10(b) and 11(b)). It can also be observed from the frequency response that there is the nonlinear coupling between the nonlinear resonance frequencies of excitation with the other two nonlinear resonance frequencies of vibration indicating the sharing of energy with other two modes. 


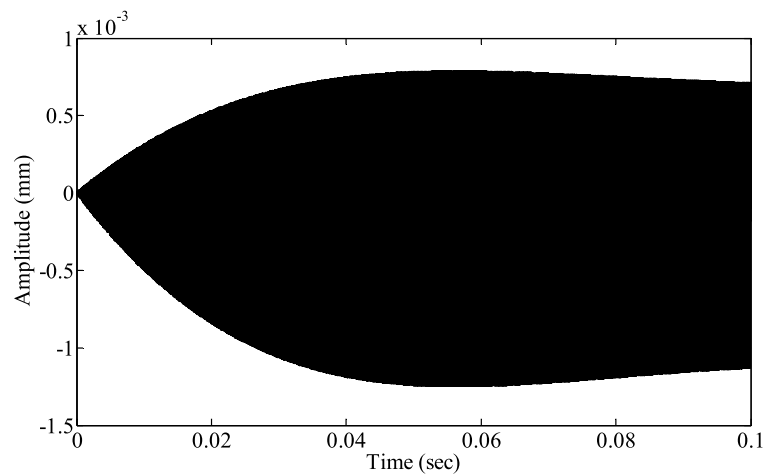

(a) Time response

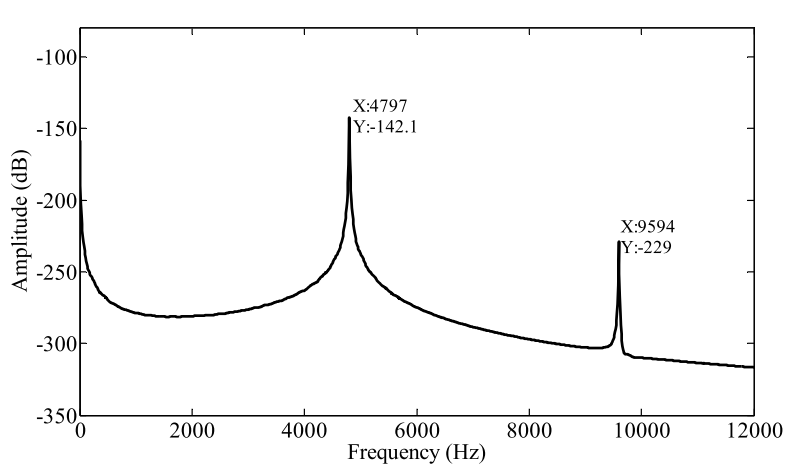

(b) Frequency response

Fig. 9. Response at first nonlinear resonance frequency of excitation.

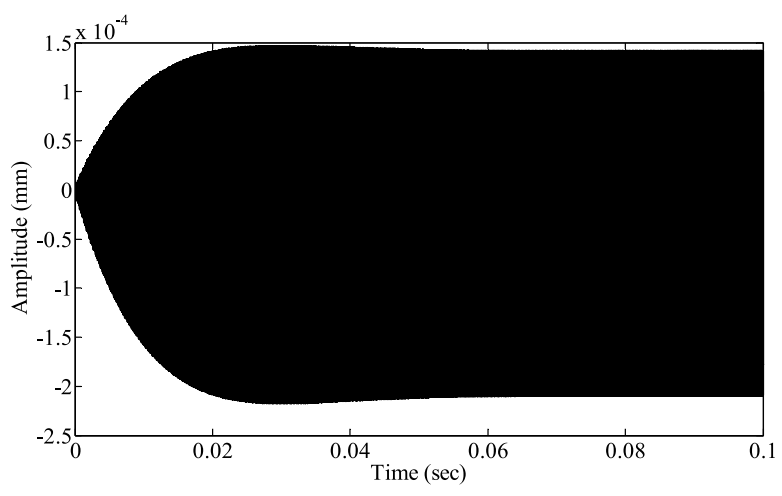

(a) Time response

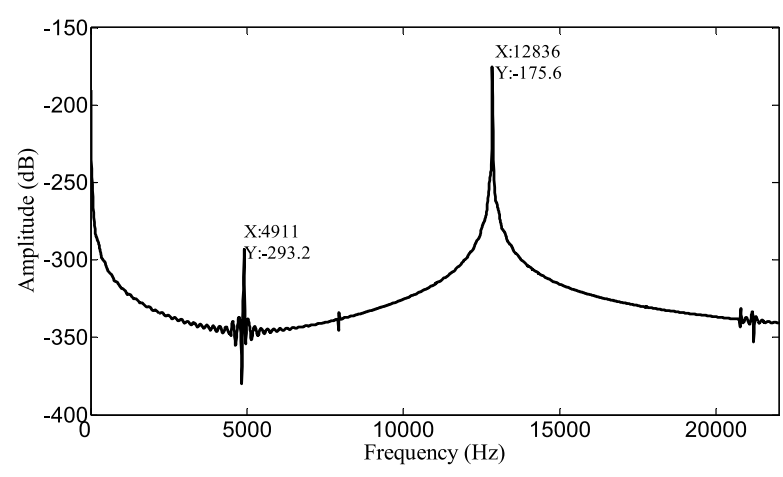

(b) Frequency response

Fig. 10. Response at second nonlinear resonance frequency of excitation.

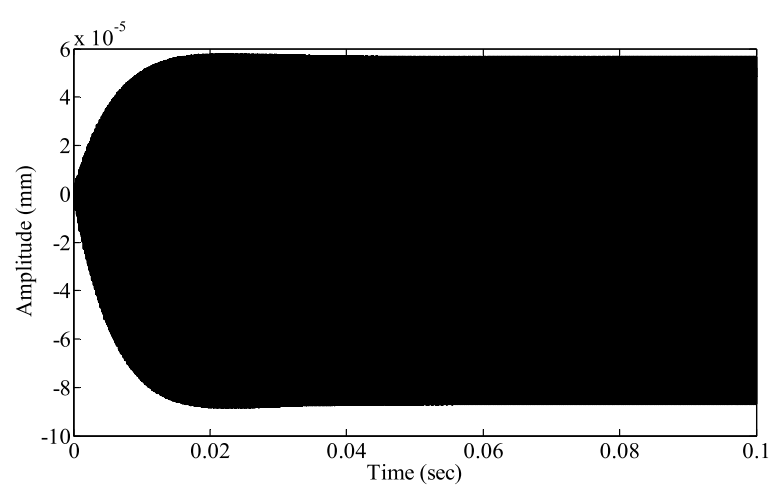

(a) Time response

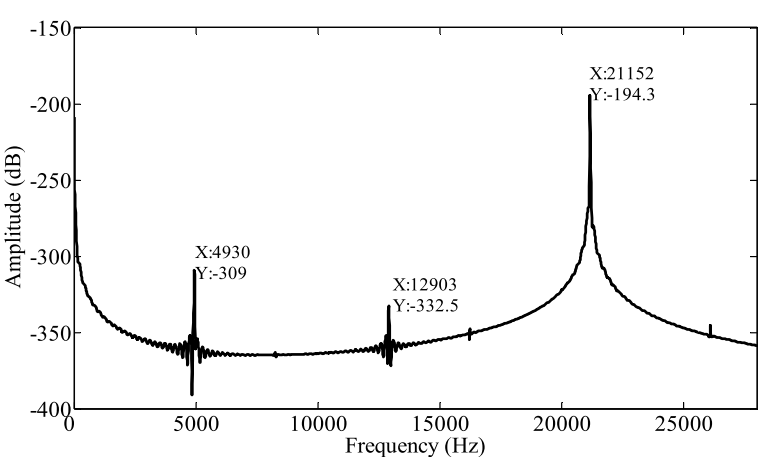

(b) Frequency response

Fig. 11. Response at third nonlinear resonance frequency of excitation.

\section{Intensity of crack on intensity of higher harmonics along bar}

The cracked bar was excited at the resonance frequencies of uncracked bar occurring at $4206 \mathrm{~Hz}, 12620 \mathrm{~Hz}$ and $21029 \mathrm{~Hz}$ to obtain the steady-state response at each of this frequency. The measurement of the response is taken 


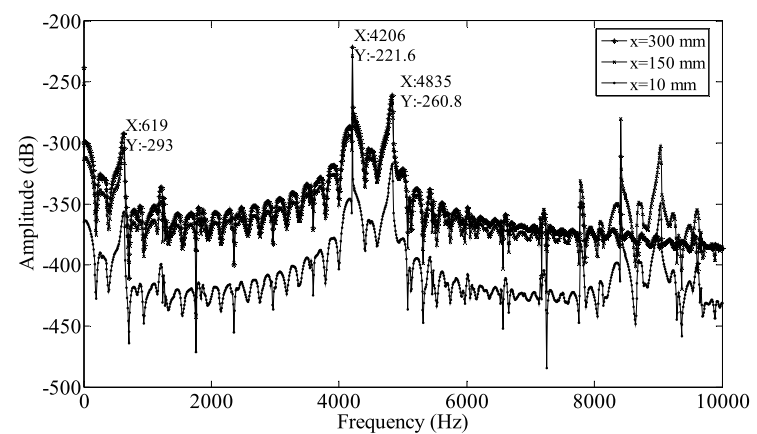

(a) First resonant frequency

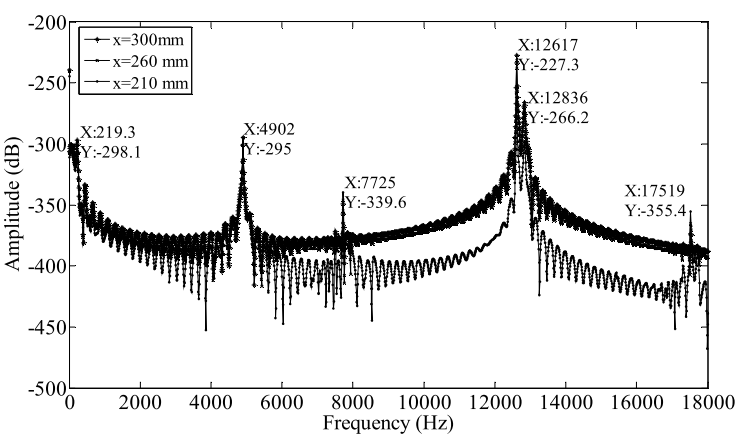

(b) Second resonant frequency

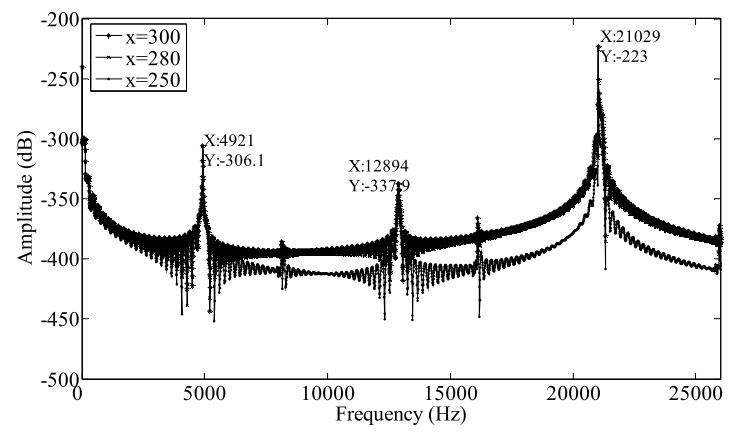

(c) Third resonant frequency

Fig. 12. Influence of measurement point from the crack on higher harmonics

at the different points on the bar, i.e. changing the distance from the crack. It was observed from the frequency response that as one moves away from the crack, the effect of nonlinearity decreases (Fig. 12).

\section{Conclusion}

The important aspect of this paper is to use the concept of dynamics compliance operator along with the theory of modal analysis to develop the Matlab-Simulimk model of the cracked bar. The methodology developed helps to reveal the complex dynamics of a cracked structure. The developed one-dimensional model of cracked bar subjected to longitudinal harmonic excitation in Matlab-Simulink can be extended to deal with multidimensional problems. It also permits implementation of analysis into automatic process for crack monitoring. This method of simulation can also be extended to ultrasonic processes.

\section{References}

[1] G. Gounaris and A. Dimarogonas, A finite element of a cracked prismatic beam for structural analysis, Computers \& Structures 28(3) (1988), 309-313.

[2] P.N. Saavedra and L.A. Cuitino, Crack detection and vibration ehavior of cracked beams, Computers \& Structures 79 (2001), $1451-1459$.

[3] G.L. Qian, S.N. Gu and J.S. Jiang, The dynamic behavior and crack detection of a beam with a crack, Journal of Sound and Vibration 138(2) (1990), 233-243.

[4] W.M. Ostachowicz and M. Krawczuk, Vibration analysis of a cracked beam, Computers \& Structures 36(2) (1990), 245-250.

[5] S. Christides and A.D.S. Barr, One-dimensional theory of cracked Bernoulli-Euler beams, International Journal of the Mechanical Sciences 26 (1984), 639-648.

[6] M.-H. Shen and Y.C. Chu, Vibrations of beams with a fatigue crack, Computers \& Structures 45(1) (1992), 79-93. 
[7] N. Pugno, C. Surace and R. Ruotolo, Evaluation of the non-linear dynamic response to harmonic excitation of a beam with several breathing cracks, Journal of Sound and Vibration 235(5) (2000), 749-762.

[8] E. Viola, L. Federici and L. Nobile, Detection of crack location using cracked beam element method for structural analysis, Theoretical and Applied Fracture Mechanics 36(1) (2001), 23-35.

[9] U. Andreaus, P. Casini and F. Vestroni, Nonlinear dynamics of a cracked cantilever beam under harmonic excitation, Journal of Sound and Vibration 42 (2007), 566-575.

[10] M. Krawczuk and W. Ostachowicz, Forced vibration of a cantilever Timoshenko beam with closing crack, Proc. Of ISMA 19, (Vol. 3), Leuven, Belgium, 1990, pp. 1067-1078.

[11] M.I. Friswell and J.E.T. Penny, A simple nonlinear model of a cracked beam, Proc. 10th Int. Modal Analysis Conf. San Diego, Ca., 1992, pp. 516-521.

[12] M. Chati, R. Rand and S. Mukherjee, Modal analysis of a cracked beam, Journal of Sound and Vibration 207(2) (1997), 249-270.

[13] A. Rivola and P.R. White, Bispectral analysis of the bilinear oscillator with application to the detection of fatigue cracks, Journal of Sound and Vibration 216(5) (1998), 889-910.

[14] V. Hiwarkar, V. Babitsky and V. Silberschmidt, Damage assessment of a cracked bar: Effect of material nonlinearity on vibro-impact response, Key Engineering Materials 413-414 (2009), 237-244.

[15] V. Babitsky, Theory of Vibro-Impact Systems and Applications, Springer, Berlin 1998.

[16] Y.G. Panovko, Internal damping during oscillation of elastic systems, Fizmatgiz, 1960 (In Russian).

[17] I.M. Babakov, Theory of vibrations. Nauka, Moscow, 1968 (In Russian). 

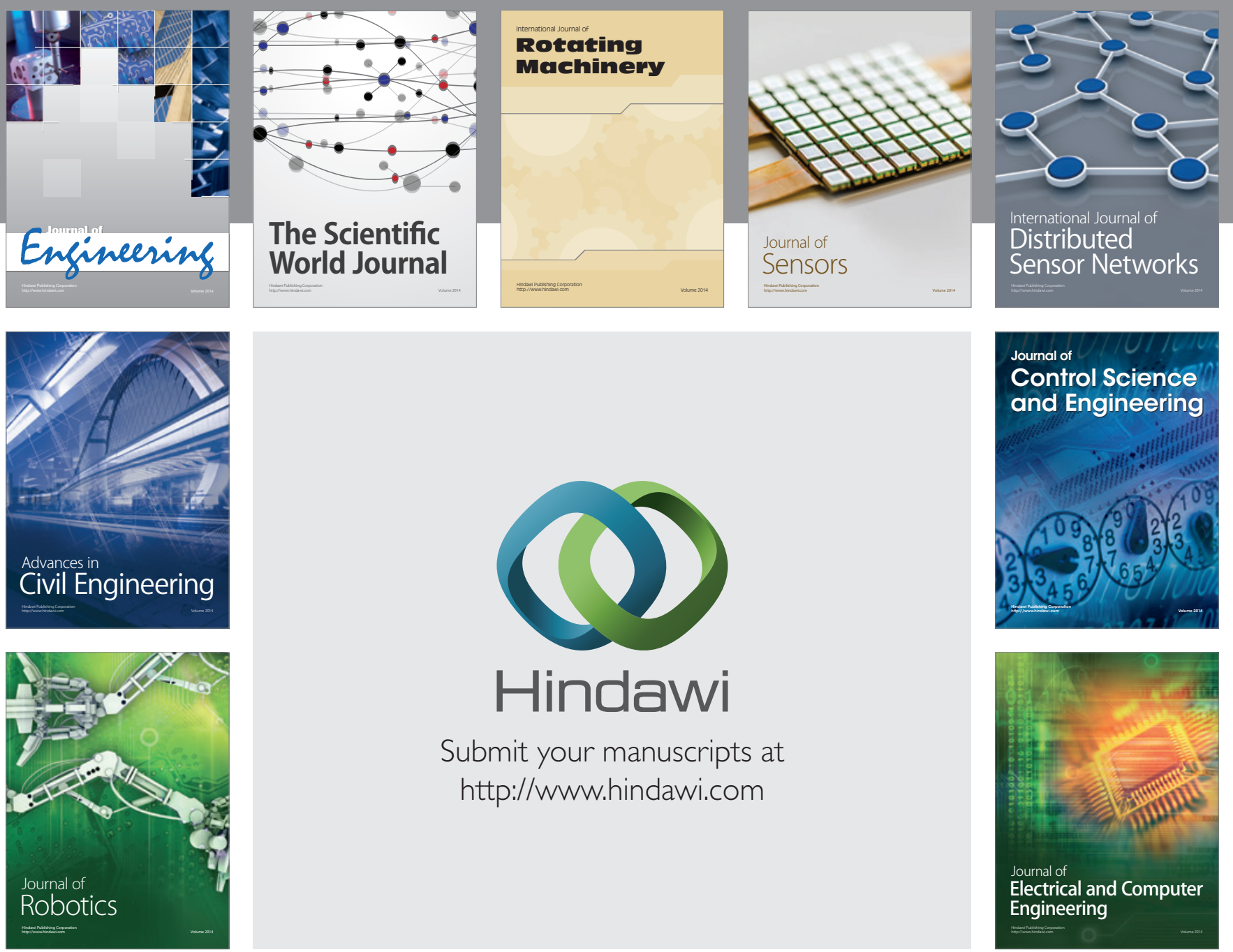

Submit your manuscripts at

http://www.hindawi.com
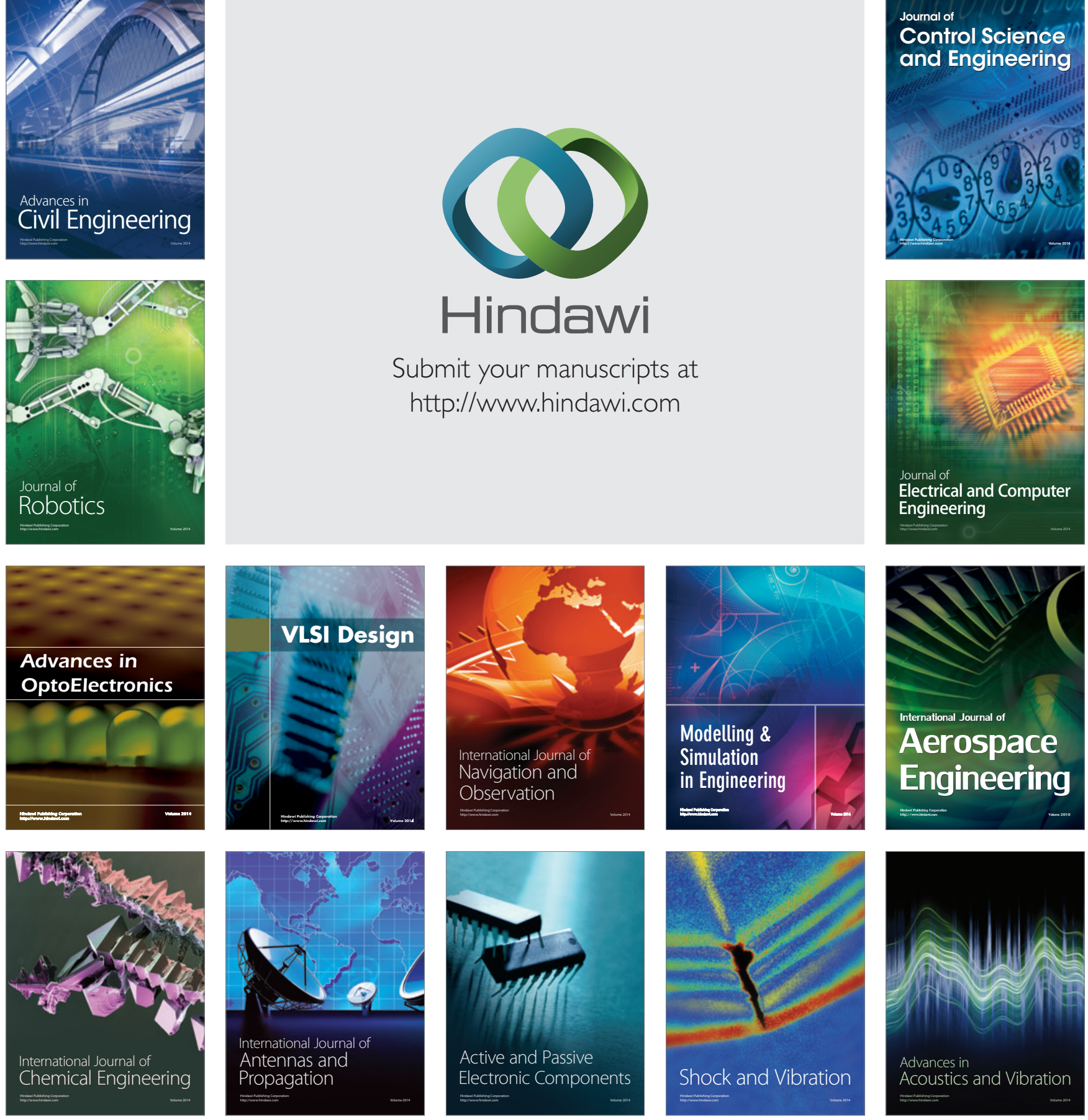